\section{Pas d'anesthésie de qualité sans des médecins anesthésistes à temps partiel}

Un des rares sujets où les leaders de droite et les leaders de gauche sont unanimes, c'est sur la nécessité pour notre Société de faire des efforts pour offrir aux professionnels des possibilités de travailler à temps partiel. Preuve en est le petit livre publié en 1997 aux éditions Mille et une nuits/Arte par Dominique Méda (philosophe français) et Juliet Schor (économiste américaine): "Travail (une révolution à venir)».

Quand on lit la vraie presse médicale - donc pas seulement ce qui a trait au TarMed ... - on trouve de nombreux arguments pour le travail à temps partiel. Je ne citerai qu'une étude récente parce que faite en Suisse par une équipe du Département d'anesthésiologie de l'hôpital universitaire de Genève: "Retentissement du vieillissement des médecins sur leur activité professionnelle: l'anesthésiologie diffère-t-elle des autres spécialités?" [1].

On y apprend, suite à l'enquête conduite, que les médecins anesthésistes ont plus de difficultés à gérer le travail de nuit que les chirurgiens et qu'ils ont une résistance à la fatigue diminuée par rapport aux chirurgiens, radiologues, internistes et généralistes. Ces anesthésistes envisagent les stratégies suivantes pour faire face au retentissement du vieillissement:

- $22 \%$ envisagent de changer de spécialité;

- $43 \%$ envisagent de changer de profession;

- $37 \%$ pensent que l'âge de la retraite devrait être imposée par la société de la spécialité;

- 73 \% envisagent de prendre une retraite anticipée;

- $45 \%$ envisagent une activité purement élective donc de ne plus faire d'urgences.
Je ne sais pas comment ils envisagent de le pratiquer dans la réalité économique actuelle des médecins.

Mais alors pourquoi voit-on des annonces dans le Bulletin des médecins suisses dans lesquelles ont met au concours un poste de médecin anesthésiste chef adjoint en précisant haut et fort que son activité doit être à temps complet? Les consœurs médecins et leur association apprécieront que la Présidente de l'Hôpital cantonal de Fribourg dont il s'agit est une Conseillère d'État socialiste.

Il est grand temps de redonner le pouvoir aux professionnels de la médecine, à des dirigeants à l'écoute des hommes et des femmes qui travaillent jour et nuit, à des responsables qui ont une vision et qui préparent les changements nécessaires à la mise en pratique des stratégies envisagées par de si fortes proportions des médecins anesthésistes ou à prévenir de telles demandes. Sans quoi la sécurité et la qualité en anesthésiologie reviendra à ce qu'elles étaient avant la deuxième guerre mondiale et il n'y aura bientôt plus de médecins anesthésistes.

Personnellement je me ferai anesthésier non pas par un médecin en formation mais par un médecin anesthésiste expérimenté, donc pas tout jeune mais "vieillissant", qui travaille à temps partiel, qui est frais et dispos et qui est mieux rétribué que ne le veut l'imposer le TarMed.

PD Dr Dominique Schwander, Villars-sur-Glâne

1 Chevalley C, Perneger T, Garnerin P, Forster A.

Retentissement du vieillissement des médecins sur leur activité professionnelle: L'anesthésiologie diffère-t-elle des autres spécialités médicales? [abstract] J Suisse Méd / Schweiz Med Wochenschr 2000;130(Suppl 123):15S. 